\title{
Stanley et Conrad, paradigmes de deux traditions discursives sur l'Afrique centrale
}

\author{
Version française de «Stanley and Conrad, Founders of alternate discourses about \\ Central Africa», -- communication présentée à l'invitation de la Sezione Colonialismo e \\ Post-colonialismo du XVIIIe Congresso scientifico dell'Associazione Italiana di \\ Anglistica (Metodi e percorsi comparatistici nelle lingue, culture et letterature di \\ origine europea). Universita degli Studi di Genova, 30 sett.-2 ott. 1996. \\ Cette version française a été présentée au Colloque L'œil de l'autre. Bruxelles, Hôtel \\ Charlier, 3-4 décembre 1996. \\ Référence de la version publiée : \\ Halen, Pierre. «Stanley et Conrad, paradigmes de deux traditions discursives sur \\ 1'Afrique centrale». In : L'Eil de l'autre. Actes des colloques de Kinshasa (9 et 10 juin \\ 1996) et de Bruxelles (1er et 2 décembre 1996). Numéro coordonné par la R.S. \\ Tshibola Kalengayi, Marc Quaghebeur, Jean-Claude Kangomba, Annick Vilain. \\ Bruxelles : Archives et Musée de la Littérature ; Kinshasa, CELIBECO, 2000, 2 vol. (A \\ \& B), en tout 648 p. (=Congo-Meuse. Revue des Lettres belges et congolaises de \\ langue française. Publication annuelle, $\mathrm{n}^{\circ} 2$ et 3, 1998/1999); vol. A, pp.63-90.
}

Le corpus littéraire et paralittéraire qui traite de l'Afrique Centrale dans des langues européennes est sans doute principalement écrit en français; il n'en comporte pas moins également des œuvres rédigées en néerlandais, évidemment, mais aussi dans d'autres langues, principalement l'anglais, l'italien, l'allemand, sans oublier les langues scandinaves et même ibériques. Ceci s'explique en partie par la très large médiatisation journalistique et/ou littéraire dont le Congo a fait l'objet, surtout mais non seulement lors des deux périodes de crise politique que furent celle du "Caoutchouc rouge », qui culmine en 1903-1904, et celle de l'indépendance, de 1959 à 1965 : une importante dimension internationale préside donc aux développements successifs de l'écriture ou plus généralement du discours (pour y inclure aussi les images, et notamment le cinéma) consacré à l'Afrique centrale. Ceci, quant à l'aspect historique. Quant à l'aspect théorique, la diversité de ces «témoins » invite dès lors à conceptualiser les approches littéraires coloniales, exotiques et post-coloniales à un niveau d'abstraction qui permette d'y inclure ou du moins d'y situer l'ensemble de ces discours (sans négliger les éventuelles retombées sur la littérature africaine). 
Sans doute était-il urgent, il y a quelques années, de travailler à un premier inventaire du corpus colonial lié à la Belgique francophone. C'est chose faite, tant du point de vue des stéréotypes et du racisme - entreprise inaugurée en 1985 par JeanPierre Jacquemin et depuis lors poursuivie -, que du point de vue des grandes configurations de l'imaginaire, auxquelles je me suis attaché dans un travail dont la partie thématique, précisément, a paru en 1993 1. D'autre part, un certain nombre de « lectures » d'œuvres particulières et de mises en perspective plus générales ont été publiées ${ }^{2}$. Enfin, les premiers matériaux historiques, par ailleurs, ont commencé à être rassemblés et, même si nous sommes très loin encore d'aucune synthèse un peu ample, on peut dire que le champ est désormais ouvert. L'objectif est à cet égard double : d'une part, il faudrait assurément que notre connaissance factuelle de l'histoire et de ses acteurs soit développée ; d'autre part, une analyse - une découpe - doit être déployée, de manière à faire ressortir les orientations distinctes que prennent des systèmes de représentations et d'intérêt qui apparaissent aujourd'hui plus complexes, plus variés, beaucoup moins unifiés qu'il semblait naguère.

Il est par conséquent plus nécessaire que jamais d'affiner les approches conceptuelles, car les outils forgés au sein de la littérature générale, s’ils sont précieux, ne sont pas toujours une technologie appropriée, ou du moins ne suffisent-ils pas à faire émerger des rhétoriques spécifiques. Par ailleurs, l'imposition de cadres idéologiques sur ce corpus est certes éclairante, mais ces cadres ne peuvent forcément pas rendre compte de l'articulation spécifiquement littéraire, ou plus largement culturelle, des termes et valeurs en discussion. Je crois avoir montré, par exemple, que le roman Kufa de Henri Cornélus avait, à un autre niveau de profondeur que celui du simple débat proou anticolonial, raciste ou anti-raciste, des dimensions complémentaires et non moins intéressantes. Cet effort d'affinage doit par ailleurs être entrepris à un niveau qui permette la confrontation de domaines - coloniaux exotiques ou post-coloniaux - qu'on a peut-être trop tendance à répartir en fonction d'une raison géographique ou linguistique qui n'a rien d'une raison épistémologique. Ainsi, on a pas mal publié sur l'Indochine ces dernières années, ou sur les complexes iliens, ou encore sur l'Amérique colombienne, sans trop se soucier du fait que ces parties du monde avaient fait l'objet de regards, sinon communs, du moins comparables. Enfin, il faudra bien un jour que les approches dites littéraires intègrent les avancées réalisées par des spécialistes des sciences sociales comme Bogumil Jewsiewicki, tant il est vrai, comme ce dernier l'a montré suffisamment, qu'on gagne à ne pas isoler les problématiques; dans la même perspective, il y a lieu désormais de formaliser les rapports entre littérature et arts plastiques.

Dans ce contexte, il me parait opportun de revenir sur un certain nombre de propositions que je formulais naguère ${ }^{3}$. Ces essais d'une première théorisation

1 «Le petit Belge avait vu grand». Une littérature coloniale. Bruxelles, Labor, 1993, 397 p., Archives du Futur.

2 On songe ici, bien entendu, aux différents volumes parus sous le titre Papier blanc, encre noire, à Bruxelles et à Kinshasa, mais aussi à d'autres parutions éparses.

3 Dans le premier volume, inédit, de la thèse défendue à l’U.C.L. en 1991. 
comportaient en effet des éléments facilement transférables à d'autres corpus que celui de la littérature coloniale belge francophone. Les travaux entrepris depuis lors ont plutôt confirmé leur portée et leur opérabilité. Par ailleurs, un essai paru en 1992 est venu leur apporter, à partir de tout autres matériaux, une manière de confirmation en même temps qu'une incitation à poursuivre la réflexion; entre autres, cet essai ouvrait de manière plus nette la perspective comparatiste qui me parait s'imposer en la matière et que je me propose de prolonger ici.

\section{La "perception du lointain"}

L'objectif reste, à mon sens, l'élaboration d'un cadre conceptuel qui permette une approche un tant soit peu rigoureuse des différents corpus « exotiques », de sorte que puissent converger des approches thématiques et rhétoriques, mais aussi historiques et identitaires ou, si l'on préfère, anthropologiques. Or l'ouvrage auquel je fais allusion est riche d'une hypothèse de travail qui me parait particulièrement féconde à cet égard ; l'auteur n'en tire pas lui-même tout le profit qu'il aurait pu en tirer, du moins s'il avait nourri des ambitions scientifiques du même ordre que les nôtres, et il ne se soucie pas non plus de la vérifier dans les textes. Il lui revient cependant d'avoir formulé une intuition qui me parait des plus éclairantes; à nous dès lors d'en tirer parti.

L'ouvrage en question, dû à Bernard Piniau, est un essai intitulé Congo-Zaïre (1874-1981). La perception du lointain ${ }^{4}$. Paru à l'époque de la Conférence Nationale Souveraine au Zaïre, tandis qu'une attention extrême était accordée aux aléas de la politique, cet essai est passé d'autant plus facilement inaperçu que son intitulé n'était peut-être pas assez explicite. Probablement disparaissait-il alors dans la masse des livres publiés depuis 1991 sur le Zaïre, - y compris chez le même éditeur L'Harmattan qui n'avait peut-être pas intérêt à dissiper le malentendu provoqué par le titre -, dont la plupart consistent en réflexions sur les destinées problématiques du pays et en bilans prospectifs. Or ce n'est pas de cela qu'il s'agit dans cet ouvrage, qui d'ailleurs était rédigé avant les événements de 1991.

Le sous-titre : La perception du lointain, suggère la portée générale de son propos : à l'aide du cas congolais-zaïrois, il s'agit de s'interroger sur la manière dont les « réalités» lointaines nous sont présentées, via un certain nombre de "médias », d'intermédiaires, de filtres déformants. A priori, Piniau ne s'intéresse pas particulièrement à la littérature, et c'est, dans la perspective adoptée ici, son mérite : son point de départ est plutôt le rôle de $C N N$ dans ce que nous avons perçu en Europe de la Guerre du Golfe, dont on se souvient qu'elle fut quasiment transformée en jeu Nintendo par la grâce des caméras. Avec une si mauvaise information, conclut Piniau, inutile de dire que les décideurs politiques évalueront mal la situation et ont toutes les chances de prendre de mauvaises décisions. Tel est l'horizon général : l'ensemble du regard avec lequel l'Occident envisage le « lointain » et l' « Autre ». Le thème est assurément à la

4 Paris, L’Harmattan, 1992, 285 p., coll. Racines du Présent. Bernard Piniau, qui fut responsable des Centres culturels français de Kisangani et de Lubumbashi, est lui-même, selon le prière d'insérer, directeur d'une association placée sous le signe du «Dialogue entre les cultures». 
mode, mais on aurait tort de ne l'envisager que dans sa dimension littéraire ou culturelle. Piniau montre qu'il est d'une importance capitale pour le devenir politique de la planète et, ce qui devient encore plus intéressant, il montre aussi le rôle que joue la littérature à cet égard.

Son ouvrage comporte essentiellement deux parties : la première est consacrée à la représentation littéraire de l'Afrique centrale, et c'est bien sûr celle-là qui retiendra toute notre attention. Mais on ne perdra pas de vue la seconde, plus longue, qui concerne le champ des communications sociales, c'est-à-dire, en l'occurrence, la manière dont les réalités du «Tiers-Monde» sont représentées par les médias européens avant de se traduire en décisions politiques souvent inadéquates. A titre d'exemple, Piniau montre avec succès comment une imagerie littéraire va progressivement remplacer l'information objective dans la presse belge (dans cinq quotidiens : Le Soir, La Libre Belgique, La Meuse, Le Peuple et Le Drapeau Rouge) à l'époque de l'Indépendance du Congo. Piniau exhibe effectivement les carences d'une information qui, à gauche comme à droite, se révèle incapable de l'objectivité (l'objet est négligé) et de la rationalité (clichés et mythes servent d'explication) qu'on était en droit d'en attendre. Ceci se répercute forcément aussi dans le discours politique de l'époque ${ }^{5}$. L'ensemble de sa démonstration, qui trouve ensuite à rebondir en France au moment des «Guerres du Shaba», est assurément convaincant. En somme, faute d'une information sérieuse, on assiste à la « victoire de l'imaginaire », à l'envahissement du champ de la décision politique par des mythes et des fantasmes "littéraires". Observons par ailleurs qu'il est somme toute assez rare de voir aussi clairement exposés les rapports éventuels entre littérature et politique, rapports qui, en l'occurrence, sont facilités par le rôle de la presse ; et, dans cette perspective, notons que, pour une fois, c'est en quelque sorte la littérature qui détermine la société, ce qui inverse le rapport traditionnel des études de sociologie littéraire.

Le livre s'inscrit donc dans le champ des proliférantes études d' « images » (images du Noir, images de l'Afrique), mais son originalité est d'étudier cette fois un cas précis dans un contexte bien délimité, et de montrer clairement les conséquences d'une désinformation globale, qui provient essentiellement d'un égocentrisme à courte vue, à la fois quantitatif (la carence des moyens requis par une information sérieuse) et qualitatif (l'informateur cherche moins à découvrir et à expliquer l'objet concerné qu'à illustrer son propre schéma de pensée ou celui de son public, quand l' " envoyé spécial » ne cherche pas seulement à justifier son voyage en présentant les choses sous un jour dramatique).

\section{L'exotisme conradien}

Venons-en à cette première partie du livre, intitulée «Le Fleuve », partie qui s'avère du plus grand intérêt pour qui s'intéresse à la présence de l'Afrique centrale dans les lettres européennes ou occidentales. Elle est consacrée à une évocation de la 
tradition conradienne dans ce domaine, thème de recherche et de réflexion qui aurait pu faire l'objet d'un ouvrage en soi, tant il est riche à la fois de cas d'espèce et de répercussions littéraires et iconographiques. L'auteur met clairement en évidence la manière dont certains " écrivains-voyageurs » réputés (un français : André Gide, deux anglophones : Graham Greene et V.S. Naipaul, un italien : Alberto Moravia) se sont en réalité promenés en Afrique centrale avec les œuvres de Conrad en tête, et parfois explicitement sous le bras. On aurait pu ajouter d'autres cas à cette série de quatre écrivains, mais l'essentiel n'est pas là.

De l'Afrique, ils n'ont vu que ce qui coïncidait avec la représentation conradienne, supposée vraie. On peut s'interroger sur ce qui fonde cette supposition de vérité : assurément, d'un côté, ces écrivains savaient que Conrad avait été, pendant une fort brève période de sa vie, capitaine d'un petit steamer sur le fleuve Congo ; ils pouvaient donc en déduire que An Outpost of Progress tout comme Heart of Darkness se fondaient sur une expérience vécue, et ils n'avaient évidemment pas tort. D'un autre côté, ces écrivains non belges étaient issus de pays ou de zones linguistiques où avaient résonné durablement l'écho de l'affaire du Red Rubber et les campagnes antiléopoldiennes de Morel; où, par conséquent, une image négative du Congo était présente, que quasiment aucun contre-discours n'était venue compenser, faute d'autre crise : lorsque nous pensons au Koweit, est-ce que nous n'avons pas immédiatement sous les yeux les dernières images que nous en avons vu sur $C N N$ et ce, bien que le pays ne soit sûrement plus dans l'état où il se trouvait lors de la Guerre du Golfe ? Quant aux représentations, le Congo était de la même manière resté dans l'état où il se trouvait lorsque les dernières images médiatiques en avaient été largement diffusées, au début $\mathrm{du} \mathrm{XX}^{\mathrm{e}}$ siècle. Enfin, des raisons plus institutionnelles interviennent, et ce sont peut-être les plus importantes: Conrad est un grand écrivain; bien que le premier traducteur en français d'An Outpost of Progress soit belge ${ }^{6}$, la France va découvrir et reconnaitre ses qualités dans le courant des années vingt, si l'on songe à Gide. Et citer un grand écrivain est toujours se grandir ; d'autant que, comme on le sait, il appartient à la rhétorique du récit de voyage de se situer toujours dans les pas d'un voyageur antérieur, de préférence célèbre ${ }^{7}$. Le Réel importe moins dès lors que la citation, l'Autre rencontré importe bien moins que le lecteur européen, et quant au plaisir de celui-ci, il est toujours augmenté lorsqu'il peut se retrouver en pays connu (dans les livres de Conrad) plutôt que de devoir apprendre de l'inconnu (les pays concernés).

Le problème, c'est que la vision de Conrad était elle-même une construction littéraire, dont l'enjeu était moins le Congo qu'un Sujet occidental en crise, sur la scène d'un théâtre auquel l'Afrique centrale fournissait un décor de déliquescence générale et, littéralement, de fin-du-monde. Luc Rasson, dans un article fondamental paru en 1988,

6 Un avant-poste de la Civilisation, traduit de l'anglais par G.D.Périer. Bruxelles, Renaissance d'Occident, 1925, 53 p. ; la traduction de G. Jean-Aubry : Un avant-poste du progrès parait chez Gallimard en 1932.

7 Cfr e.a. le bel ouvrage de Sarga MOUSSA : La relation orientale. Enquête sur la communication dans les récits de voyage en Orient (1811-1861). Paris, Klincksieck, 1995, 279 p., coll.

Littératures des voyages $\mathrm{n}^{\circ} \mathrm{IX}$. 
avait déjà pointé les ambigüités de la représentation conradienne, dont la réputation anticolonialiste est en réalité très usurpée ${ }^{8}$. On pourrait ajouter à cette approche limitée à la littérature l'apport ultérieur du cinéma, les trois films qui, à ma connaissance, ont été consacrés à adapter Heart of Darkness pour l'écran et le petit écran ayant tous souligné l'aspect initiatique et anthropologique, voire mystique, du récit: on se souvient e.a. des images d'Apocalypse Now et de la destinée de Fr. F. Coppola pendant le tournage ${ }^{9}$. Heart of Darkness, comme ce film, repose d'ailleurs sur un canevas typique de l'imaginaire colonial: le mythe de l'aventurier-roi, qu'on trouve chez Malraux, chez Lawrence et chez bien d'autres écrivains plus ou moins inspirés par une gêne profonde et en même temps une fascination pour les émules de Robinson Crusoé 10 .

Bernard Piniau ne cite pas cet article ni ce film, pas plus qu'il ne cite d'ailleurs aucun autre travail ${ }^{11}$. Son propos est à la fois utilitaire (il s'agit de préparer la seconde partie de l'ouvrage) et plus intuitif que systématique. Cela ne l'empêche pas d'être convaincant, sauf peut-être quant à la délimitation de l'objet thématique lui-même : «Le Fleuve ». En effet, la tradition conradienne se retrouve aussi bien, par exemple, dans le Voyage au bout de la nuit de Céline, qui n'évoque pas le Congo, et d'une autre manière, bien plus tard, dans Le Reste du monde d'Anna Geramys, dont l'action se déroule au bord du Tanganyka au début des années 70. L'important est plutôt dans l'élaboration progressive de ce lieu-commun : une Afrique mystérieuse, dangereuse et primitive, essentiellement rétive -- selon le concept précieux qu'a proposé Luc Rasson - aux efforts prométhéens de la construction historique et d'un certain humanisme.

Mais il est vrai que le Fleuve Congo est par excellence le théâtre de cette mise en échec : c'est la voie symbolique d'une remontée du Temps, la traditionnelle image du cours d'eau héraclitéen faisant place en l'occurrence à un contraste saisissant entre d'une part la petitesse et la vanité de l'effort européen pour le dominer, pour en avoir raison, et d'autre part le gigantisme quasi métaphysique d'un courant et d'un décor qui vouent cet effort à l'anéantissement. Le voyage se déroule moins dans un espace moins

8 RASSON (Luc), «"Chacun sa place". L'anticolonialisme dans Heart of Darkness (1899) et dans Voyage au bout de la nuit (1932)», dans BUISINE (A.) et al. (éd.). L'Exotisme. Paris, Diffusion Didier-Érudition, 1988, 468 p., Cahiers CRLH-CIRAOI n5 ; pp.267-280.

Le film Apocalypse now, réalisé par Francis Ford Coppola avec Marlon Brando et Martin Sheen et tourné au Vietnam, avait été suivi d'un film-mémorial, Au coeur des ténèbres, réalisé par l'épouse de Coppola à partir des images du tournage de Apocalypse now. Plus récemment, l'anglais Nicolas Roeg a proposé Au coeur des ténèbres (1994) avec Tim Roth et John Malkovitch.

Robinson Crusoé, on le sait, est une projection imaginaire de l'entreprise coloniale anglaise : l'indigène étant supposé inexistant, on peut rebâtir utopiquement un modèle de développement européen où seraient aussi niées les relations de travail. Le mythe de l'aventurier-roi rétablit la présence de l'indigène, mais ne peut le faire que colonialement, c'est-à-dire sous la figure du barbare provisoirement dominé (cfr les travaux de Gilbert Soubigou). sait pourquoi, Ngando de Lomami Tshibamba ainsi que des ouvrages de Joseph-A. Cornet sur l'art africain. 
géographique que mythique: on va vers l'Origine ou vers la Fin des Temps. Assurément, le petit steamer n'est pas n'importe quel symbole : il évoque de manière plus précise la doxa positiviste et technicienne qui triomphe à la fin du XIX ${ }^{\mathrm{e}} \mathrm{s}$., et qui remplit la philosophie bourgeoise d'un Auguste Comte d'un optimisme auquel deux guerres mondiales, Auschwitz et Hiroshima, règleront son compte. Relevons déjà que, dans l'imagerie qui entourera les récits de Stanley, le petit steamer occupera une place de choix, mais avec des valeurs inverses, de conquête, de pénétration réussie, de prouesse technologique et d'exploit humain : soit la réitération de Robinson Crusoé, hors l'espace abstrait de l'île. C'est, dans Through the Dark Continent et dans The Grounding of the Congo Free State, le Lady Alice et, plus significatif encore, L'EnAvant. Le steamer de Stanley symbolise précisément cette doxa positiviste dont se nourrit plus largement toute l'utopie coloniale: ne rêve-t-elle pas d'une sorte de transparence du monde à lui-même, transparence qui se réaliserait par une double avancée dans le Temps (le Progrès) et dans l'Espace? En traversant l'ex-terra incognita, l'explorateur montre que la zone n'est pas opaque, mais plus que simplement pénétrable : nommable et «ouverte», selon le leitmotiv de l'époque, aux commerces divers de l'Histoire. Ainsi le Belge de Lichtervelde peut-il placer sous une épigraphe de Térence (humanum sum et nil humanum a me alienum puto) un essai consacré aux Légendes de l'inconnu géographique, qu'il conclut ainsi, en 1903 :

A l'heure présente, l'ingénieur forge les derniers rails qui, du Cap Horn aux glaciers de l'Alaska, et des mers d'Extrême-Orient au Cap de Bonne-Espérance, doivent former l'anneau des épousailles de l'homme et de la terre. Par la colonisation, la jeunesse aventureuse décuple les champs ouverts à son activité, la lutte pour la vie se transforme en expansion nationale, le prolétaire fonde une famille, le convict redevient un citoyen, le déclassé se relève, l'individualisme triomphe du collectivisme, la richesse se féconde, le monde s'unifie, les préjugés s'effacent, la tolérance entre dans les coeurs et graduellement le sauvage cannibale, l'esclave héréditaire s'élève, grâce à d'augustes et généreuses initiatives, au niveau des races dominantes ${ }^{12}$.

Cette citation est éclairante : on y trouve l'ethnocentrisme et les stéréotypes racistes, bien entendu, mais là peut-être n'est pas l'essentiel puisqu'ils ne sont pas fondamentalement liés à un mouvement qui, précisément, vise à «effacer les préjugés ». Plus importante est l'appréhension coloniale-prométhéenne de l'espace à connaitre, qui hante à l'époque, dans tous les pays européens, les fameuses Sociétés de Géographie où s'élaborent aussi, il faut le rappeler, les premières «missions humanitaires », notamment anti-esclavagistes.

C'est très exactement contre cette conception que s'érige la tradition conradienne, aussi appelée «exotisme critique». Elle doit être comprise moins comme un témoignage à propos de l'Afrique coloniale et post-coloniale, que comme une littérature de crise, vouée à illustrer la faillite ou l'inopérabilité de l'humanisme et du positivisme matérialiste. En ce sens, on peut aussi qualifier cette tradition d'« exotisme radical», tant il est vrai que tout y est construit à partir d'un postulat, celui de l'altérité. Non pas

12 DE LiChterVelde (G.), Les Légendes de l'inconnu géographique. Bruxelles, Lacomblez, 1903, 82 p. ; ici pp.80-81. 
le Divers à la Segalen, et encore moins l'Autre tel que le conçoit le discours du « respect des différences » et du « dialogue entre les cultures ». Mais l'Autre essentiel, d'ordre métaphysique plutôt qu'humain : cet illimité qui marque la limite du Sujet ${ }^{13}$.

La cuvette congolaise devient ainsi le théâtre emblématique d'un drame, celui de l'anéantissement de l'Histoire, indifféremment attirée tantôt vers son Origine magmatique, tantôt vers sa Fin chaotique. D'où un certain nombre de topoï secondaires : le fleuve lui-même, qu'on remonte en remontant aussi le Temps ; la forêt équatoriale, ce mur opaque et impénétrable, cet enchevêtrement indifférencié ${ }^{14}$, fait d'une végétation exubérante et/ou pourrissante ; le «sauvage », incompréhensible ou animal ; le « colonial », ce Prométhée déchu et médiocre autant qu'inepte ; l'ensemble formant l'univers de la non-Histoire, dont aucune évolution n'est à attendre, par définition, puisque c'est sur lui que l'Histoire vient buter et se révéler vaine. C'est le lieu mythique où l'être peut s'apercevoir d'une altérité qui le gagne, qui lui révèle d'abord l'altération de ses facultés, enfin son aliénation : s'il ne quitte pas l'endroit, il est voué à la mort ou à la folie. Cette déliquescence est exemplairement montrée par le suicide plus ou moins volontaire (la volonté du Sujet doit s'annuler) du protagoniste de $K u f a$, roman de Henri Cornélus (1954), récemment réédité : celui qui aurait dû être le héros d'une Histoire de libération pour l'Afrique s'annihile par noyade au milieu $d u$ centre, si l'on peut dire, tant il est vrai qu'il s'agit de se perdre au milieu de «l'Eau noire », cette mer (mère ?) intérieure de la cuvette congolaise (le « bassin »), elle-même au centre du continent ${ }^{15}$. Retourner à cette mère, s'y engloutir, renoncer à la responsabilité historique du langage, rêver de se perdre dans le magma originaire : c'est l'envers du discours prométhéen et colonial ${ }^{16}$.

Le paysage conradien, en réalité, utilise le référent de l'Urwald pour parler de bien autre chose que de l'homme en Afrique; on qualifierait difficilement, du reste, son point de vie d'humaniste. Ainsi, les deux protagonistes blancs de An Outpost of Progress :

They lived like blind men in a large room, aware only of what came in contact with them (and of that only imperfectly), but unable to see the general aspects of things. The river, the forest, all the great land throbbing with life, were like a great emptiness. Even

13 Cfr MiCHEL (M.), «Figures de l'exotisme et désir d'au-delà», dans R. ANTONIOLI (éd.), Exotisme et création. Lyon, Publications de l'Université Jean Moulin - L'Hermès, 1985, pp.345355.

Cfr nos propositions : «L'Ouvert et le Fermé : une typologie de l'espace centre-africain dans toutes sortes d'histoires européennes», dans Descriptions et créations d'espaces dans la littérature. E. Léonardy et H. Roland (éd.). Bruxelles, Nauwelaerts, 1995, pp.215-233 ; «Paysage exotique et paysage colonial», dans Le Paysage et ses grilles. Actes du colloque de Cerisy. Textes réunis et présentés par Françoise Chenet. Paris-Montréal, L’Harmattan, 1996, pp.51-70. Voir notre introduction, «A travers le continent rétif», à La littérature coloniale, II. Bruxelles, Le Cri, 1995, coll. Les évadés de l'oubli ; et «Le petit Belge avait vu grand». op.cit., pp.187-246. coloniale française, mais aussi chez d'autres écrivains comme Paul Bowles. Voir son ouvrage : L'Orient voilé. Paris, Zulma, 1993, 297 p. 
the brilliant sunshine disclosed nothing intelligible. Things appeared and disappeared before their eyes in an unconnected and aimless kind of way. The river seem to come from nowhere and flow nowither. It flowed through a void. Out of that void, at times, came canoes, and men with spears in their hands would suddenly crowd the yard of the station. They were naked, glossy black, ornamented with snowy shells and glistening bass wire, perfect of limb. They made an uncouth babbling noise when they spoke, moved in a stately manner, and sent quick, wild glances out of their startled, neverrestling eyes. Those warriors would squat in long rows, four or more deep, before the verandah, while their chiefs bargained for hours with Makola over an elephant tusk. Kayerts sat on his chair and looked down on the proceedings, understanding nothing. He stared at them with his round blue eyes, called out to Carlier, "Here, look at that fellow there -- and the other one, to the left. Did you ever see such a face ? Oh, the funny brute !"

Carlier, smoking native tobacco in a short wooden pipe, would swagger up twirling his moustaches, and surveying the warriors with haughty indulgence, would say --

"Fine animals. Brought any bone? Yes ? It's not to soon. Look at the muscles of that fellow -- third from the end. I wouln't care to get a punch on the nose from him. Fine arms, but legs no good below the knee. Couldn't make cavalry men of them" [...]

Such profitable visits were rare. For days the two pioneers of trade and progress would look on their empty courtyard in the vibrating brilliance of vertical sunshine. Below the high bank the silent river flowed on glittering and steady. On the sands in the middle of the stream, hippos and alligators sunned themselves side by side. And stretching away in all directions, surrounding the insignificant cleared spot of the trading post, immense forests, hiding fateful complications of fantastic life, lay in the eloquent silence of mute greatness. The two men understood nothing, cared for nothing but for the passage of days that separated them from the steamer's return 17 .

On aperçoit le procédé de saturation qualificative auquel a recours Conrad, en même temps que l'enjeu ontologique d'une telle mise en œuvre. En particulier, l'adjectif «fantastique » rend visible les affinités d'un tel exotisme avec un état d'esprit de résistance à l'égard d'une certaine rationalité positiviste, état d'esprit qu'on observe sans difficultés dans d'autres productions littéraires européennes de l'époque.

Inutile d'y insister beaucoup: la tradition conradienne est de toute évidence dominante lorsqu'il s'agit d'évoquer l'Afrique centrale pour des lecteurs (des auditeurs, des spectateurs) européens. Et, fait intéressant, on la retrouve à la fois dans les œuvres du champ légitime et du champ illégitime, ce qui est un bon indice qu'on a bien là une représentation collective où l'ensemble de la société occidentale se donne le plaisir trouble de décrire son Autre : non pas, en réalité, l'homme Africain, auquel personne ne s'intéresse, d'autant qu'il pourrait bien rappeler le lecteur à ses propres problèmes humains ou encore à sa responsabilité historique vis-à-vis de lui, mais ce que l'Occidental veut penser que lui-même n'est pas : l'envers de son projet historique et de la conscience de lui-même qu'il veut avoir. Ainsi, l'Urwald apparait dans les premières lignes du best-seller délirant de Michael Crichton, l'auteur aussi de Jurassic Park, comme « le lieu où se trouvent les os »:

17 Complete Work, vol. VIII : Tales of Unrest. Kent Edition. New York, Doubleday, Page \& Cy, 1925, pp.92-94 ; Trad. française : cf. Un avant-poste du progrès, dans Inquiétude. Euvres. T.I. Paris, NRF/Gallimard, Bibliothèque de La Pléiade, 1982, pp.728-730. 
L'aube se leva sur la forêt humide.

Le pâle soleil consumait la fraicheur matinale et la brume à la moiteur tenace, révélant un monde gigantesque et silencieux. D'énormes arbres aux troncs de douze mètres de diamètre s'élevaient à soixante mètres au-dessus du sol, où ils étalaient une dense voute feuillue qui masquait le ciel et d'où l'eau dégouttait perpétuellement. Des rideaux de mousses grises, de plantes grimpantes et de lianes pendaient des arbres en s'enchevêtrant; des orchidées parasites jaillissaient des troncs. Au niveau du sol, d'immenses fougères luisantes d'humidité croissaient à hauteur d'épaule et retenaient le brouillard à ras de terre. Çà et là, une tache de couleur : les rouges efflorescences des acanthemas, poison mortel, et les fleurs grimpantes bleues des dicindras qui ne s'ouvraient qu'au petit matin. Mais on avait surtout l'impression d'un monde vaste, démesuré, d'un vert teinté de gris : un milieu étranger à l'homme, inhospitalier.

Jan Kruger posa son fusil près de lui $[\ldots] 18$

Quant à savoir pourquoi triomphe la vision conradienne plutôt que la vision positiviste, cela revient peut-être à se demander pourquoi nous écrivons ou nous lisons de la littérature : pourquoi nous réservons un espace à l'évocation de notre Autre, de ce qui nous menace symboliquement, du Monstre ${ }^{19}$. Du Monstre dont on triomphe, par héros interposé, ou dont on ne triomphe pas, mais qui ne sortira quand même pas du livre. C'est ce que Charles Grivel appelle la « production de l'intérêt romanesque ».

Tout ceci permet de préciser la notion d'exotisme critique. Après bien des tâtonnements, j'avais été amené à définir l'exotisme, en dehors de toute dimension géographique, comme le regard au moyen duquel on faisait d'un certain objet référentiel un Autre, c'est-à-dire parfois un simple envers (inversum), parfois un ethnostéréotype fonctionnel, isolé dans sa «différence», mais parfois aussi, et plus fondamentalement, un donné à la fois inconnu et inconnaissable, c'est-à-dire la figure de ce qui résiste à l'appropriation autant qu'à la communication. L'Afrique de Conrad, par là, est éminemment exotique. Mais il y a un autre débat. Lorsqu'on a parlé d'exotisme critique, sous l'influence de Segalen et d'une autre doxa, celle du respect des différences inspirée par l'anticolonialisme, on a voulu voir chez Conrad (comme chez Gide ou chez Céline... ou chez Cornélus) un exotisme acceptable dans la mesure où il aurait brossé le portrait de «coloniaux» un peu débiles, et, dès lors, dans la mesure où il aurait fait la critique de l'entreprise coloniale. Une telle valorisation est complaisante et ambigüe, car elle fait silence sur le fait que l'image de l'Africain est encore plus péjorative que celle des pauvres prométhées déchus au bord du Congo. En réalité, s'il y a un anticolonialisme chez Conrad, il n'est qu'une conséquence d'une critique bien plus profonde, touchant à la perspective même du progrès historique et de la possibilité de communiquer entre les humains.

18 CRIChTON (M.), Congo, New York, Knopf, 1980 ; Congo. Traduit de l'américain par J.-P. Martin (1981). Paris, Mazarine, 1982, 477 p., coll. Le Livre de Poche n`5667, p.15. Les quelques touches «scientifiques» de la description, non seulement cautionnent ici la vraisemblance, mais ajoutent à l'altérité du lieu des sèmes mystérieux, à rebours de la connaissance antexotique qu'elles auraient pu introduire. 
Ceci explique aussi la contradiction qu'on voit chez Gide, Simenon ou chez Cornélus : d'une part ils suivent plutôt généreusement un impératif humaniste, et ils dénoncent les injustices du système colonial ; d'autre part, ils ne peuvent s'empêcher de suivre la tradition conradienne d'un prodigieux mépris pour l'Afrique ou, plus précisément, d'une projection sur l'Afrique de leur propre désir de fréquenter la Sauvagerie ${ }^{20}$.

\section{La tradition de Stanley}

Avec beaucoup d'à-propos, Bernard Piniau montre aussi comment, à la fin du XIX siècle, un autre discours dispute au discours conradien la prépondérance, en Europe, au sujet de l'Afrique équatoriale : celui de Stanley. Ce discours, qui fonde la tradition que nous appelons antexotique, est un discours de maitrise et de connaissance: l'exploration géographique. Un exemple, tiré de Through the Dark Continent, et plus spécialement de la 3e partie, qui marque l'arrivée dans ce qui n'est pas le «cœur des ténèbres ». Le passage, intitulé : «Suspense and Success» et «Among friendly Natives », commence avec les précisions d'usage dans les carnets de l'explorateur ${ }^{21}$ :

"February 8. -- Thank God! An anxious day has terminated with tranquillity to longdisturbed minds. We are camped in a small jungle-covered islet in north latitude $\mathrm{I}^{\circ} 40^{\prime}$ $44^{\prime \prime}$ by observation, and east longitude $21^{\circ} 4^{\prime}$ by acct. Opposite, at 500 yards' distance, on the left bank, is the village of Rubanga, in Nganza. On the right bank, at 1700 yards' distance from us, is the large town of Gunji.

S'ensuit le récit de la «pacifique conquête » de la ville, qui se termine sous le signe de l'abondance des échanges communicationnels et matériels :

[...] The old chief nodded with his head. We raised our anchor, [...], and, snatching a string or two of cowries, I sprang on land followed by the coxswain Uledi, and in a second I had seized the skinny hand of the old chief, and was pressing it hard for joy. Warm-hearted Uledi, who the moment before was breathing furiously hate of all savages, and of the procastinating chief in particular, embraced him with a filial warmth. [...] in an incredibly short time, the blood-brotherhood ceremony between the suddenly former friends was solemnly entered into, and the irrevocable pact of peace and friendship and good-will had been accomplished ! [...] We distributed presents to each native, and in return we received great bunches of mellow, ripe, and green bananas, as well as of fish. It was agreed between us that we should encamp on this little islet, on which we find ourselves to-night, with a feeling as though we were approaching home. [...]

20 Sur Gide, voir SÄNDIG (Brigitte), «Die Macht des Klischees. Zur großen Zeit des Kolonialismus in der französischen Literatur», in Grenzgänge, 1. Jhg. 1994, heft 2, pp.81-92 ; PORRA (Véronique), L'Afrique dans les relations franco-allemandes entre les deux guerres. Frankfurt a.M., Iko-Verlag, 1994, 299 p. Nous n'avons pas pu consulter l'ouvrage dont la parution était annoncée pour cet été : Russel WEST, Conrad and Gide. Translation and Intertextuality. Amsterdam-Atlanta, Rodopi, 1996, ca. 250 p. 
Un tel discours se retrouvera dans la plupart des approches coloniales au sens propre, approches dont on sait qu'elles furent essentiellement documentaires, et toujours d'une certaine ambition ou prétention scientifique. Conrad, qui fonde l'«exotisme critique», vise au contraire, d'emblée, à la méconnaissance, puisque ce qui l'intéresse, c'est l'inconnaissable (ou ce qu'il veut supposer tel), de préférence violent et barbare, ou alors pourrissant et chaotique. Or, de cette rivalité, c'est indiscutablement Conrad qui sort vainqueur en Métropole : c'est lui qui intéressera les belles-lettres légitimées (mais aussi bien, ajoutons-nous, la paralittérature aventureuse, soient les deux voies du succès éditorial; ainsi que le cinéma) ${ }^{22}$. Piniau suggère aussi que les anthologies et les ouvrages de référence, lorsqu'ils citeront néanmoins Stanley, privilégieront dans son œuvre abondante les passages «exotiques» (dangers, combats, difficultés, sauvageries), et laisseront de côté les passages «antexotiques", pourtant plus nombreux et plus caractéristiques (mesures du terrain, acquis pratiques et géographiques, tout ce que Piniau appelle le « savoir réaliste »).

Quant à la tradition de Stanley, elle se retrouve dans la littérature proprement coloniale, dont l'impact fut réduit en métropole et que ses postulats naturalistes, liés à une philosophie de l'histoire et à une confiance dans le langage qui étaient à l'honneur à la fin du XIXe siècle, empêchaient de prétendre à une quelconque "modernité ». Ceci explique en partie pourquoi les littératures coloniales dans leur ensemble, sauf sans doute en Angleterre, ont été tenues à l'écart de la reconnaissance et de la légitimation : de la littérature, le lecteur métropolitain n'attendait pas qu'elles viennent lui rappeler les principes qui régissent le plus profondément le développement des sociétés occidentales depuis la Renaissance ; au contraire, il attendait, il attend sans doute encore, qu'elle lui montre son Autre. D'où, aussi, le caractère indécrottable, en dépit de tous les efforts entrepris en vue de représentations plus «correctes», des stéréotypes qui caractérisent jusqu'à aujourd'hui la littérature - le cinéma, la bande dessinée, le polar, etc.-, la plus lue à propos de l'Afrique. Et, de là, la presse écrite et télévisuelle.

\section{De l'exotisme post-colonial}

Rien n'a dès lors changé dans ce qui fait, globalement, notre perception de l'Afrique. Assurément, d'une part, il est question de développement, d'aide au développement, de dialogue entre les cultures, d'aide humanitaire et de partenariat. L'africanistique aussi s'est développée, comme discours scientifique. Nous en savons plus, c'est évident, et le lointain s'est rapproché, conséquence du siècle de contacts qu'ouvrait, ci-dessus, la citation de G. de Lichtervelde. Et néanmoins, d'autre part, dès que possible, nous retournons, pour notre jouissance, à la tradition conradienne : une famine, un coup d'état, une guerre civile, les tragédies ne manquent pas pour que fasse intrusion, d'un côté, dans les représentations «littéraires », de l'autre côté, dans les

22 Le cinéma et la bande dessinée sont partagés, de façon analogue, entre les deux traditions. La gravure, fin du XIX ${ }^{\mathrm{e}}$ s., le dessin scientifique et, dans la propagande des années 1950 particulièrement, la photographie en noir et blanc s'inscrivent par contre dans un dessein stanleyien (cfr «L’Ouvert et le Fermé...», art.cit.). 
médias, un imaginaire de catastrophe dont Apocalypse Now est la réalisation exemplaire (à cet égard, il importe peu que ce film ait été tourné au Vietnam). V.S. Naipaul et Moravia sont les écrivains post-coloniaux qu'évoque Bernard Piniau. Il y en a beaucoup d'autres, y compris, dans la littérature zaïroise elle-même, puisqu'on ne s'explique pas tout à fait un roman comme Cannibale de Bolya ${ }^{23}$ (placé sous une épigraphe de Conrad) sans en référer à cette tradition qui n'est donc plus strictement européenne. Le Reste $d u$ monde, d'Anna Geramys ${ }^{24}$, splendide roman qui a pour cadre le génocide hutu de 1972-73 au Burundi, est à mon sens l'exemple canonique de ce que devient l'exotisme critique dans la littérature post-coloniale, (c'est-à-dire dans un contexte où le catastrophisme, naguère plus ou moins jugulé par le discours antexotique de la « colonie modèle », peut se revendiquer de quelque justification réaliste et en même temps déployer hyperboliquement la crise historique de l'homme blanc) : la reprise d'un mythe aux dimensions métaphysiques, celui de l'anti-prométhée. Comme chez Conrad, on y assiste à la déroute de l'entreprise occidentale de progrès historique, déroute dont font les frais, par centaines de milliers, les cadavres au bord de la route, dans la rivière Akagera et dans les camps, mais dont le héros occidental se tire, finalement, sans trop de mal, sinon une neurasthénie galopante et une vague envie de s'auto-détruire 25 .

Bernard Piniau formule, en outre, une dernière suggestion: la mythification de l'Afrique littéraire et médiatique serait proportionnelle à l'abandon progressif d'un projet historique concernant la zone de référence (p.260). En quelque sorte, l'action et la possibilité d'une action, en quelque sens que ce soit, commandent l'information; l'impossibilité pratique ou politique d'intervenir ou (d'agir avec) commande la désinformation et l'irruption de fantasmes, le recours à une tradition mythique et littéraire dont il importe peu qu'elle concerne un objet donné, puisque le message est désormais non référencié. Ceci, qui parait relever du bon sens, est de nature à expliquer bien des choses, à commencer par le désintérêt avéré de la classe politique belge à l'égard de la politique congolaise, dont la nation éprouvait en profondeur qu'elle n'avait que faire. Mais aussi, en 1960, la brutale enflure médiatique du moment qu'il allait se passer quelque chose - pourvu que ce soit un peu sanglant!? - et puis le détournement de l'information vers le fantasme, du moment qu'il s'avérait que les acteurs européens étaient relativement impuissants à changer l'Histoire.

Le roman de Graham Greene, A Burnt-Out Case, vient à l'appui de cette hypothèse, puisque son action se situe explicitement entre les émeutes de Léopoldville et l'Indépendance congolaise, c'est-à-dire à l'époque où l'entreprise prométhéenne de la « colonie modèle » tourne en eau de boudin. Querry, qui n'est pas pour rien architecte, vient en Afrique à la recherche du bout du monde et de ce qu'on doit appeler le bout de

23 BOLYA, Cannibale. Ed. P.-M. Favre, 1986, 191 p. Voir ci-contre l'étude de S. Riva.

24 GERAMYS (Anna), Le reste du monde. Paris, Mazarine, 1987, 316 p. ; voir aussi son second roman, Les fruits tropicaux. Roman. Lausanne, L’Age d'Homme, 1989, 157 p. 
lui-même : "I have comme to an end of all that too. / Too ? / Like all the rest. To the end of everything " ${ }^{26}$. Sans doute, l'au-delà, le Paradis appelé Pendélé par son alter ego, le burnt-out case nommé Deo Gratias, n'est-il pas rejoint : si la charité émerge finalement comme le seul élément sûr au terme du récit, c'est une charité est aussi inefficace que tragique, qui nous éloigne peu de Conrad. Greene nous en prévient: «This Congo is a region of the mind» (p. 5). C'est la pointe extrême de l'ici-bas : «I've come to an end. This place, you might say, is the end. Neither the road nor the river go any further » (p. 110). Que le sujet historique, hérité des Lumières, y soit mis en question, c'est évident dès la première ligne : "The cabin-passenger wrote in his diary a parody of Descartes: "If feel discomfort, therefore I am alive", then sat pen in the hand with no more to record» (p.9; cf. aussi p.186). L'Au-delà, le Pendélé, prend aussi un aspect historique: on annonce l'indépendance du Congo dans cette perspective. Mais il est clair que, dès 1960, année de publication du roman, cette indépendance n'est pas le moins du monde envisagée comme une issue progressiste, au contraire, et la maladie qui affecte à la fois le décor, - décrit en termes conradiens : indifférenciation, immobilité, engloutissement, etc. - et l'être est une lèpre sans espoir de salut. Le « steamer blanc» avance, mais dérisoirement : «A lot of effort it seemed for so slow a progress » (p. 10). L'odeur de l'Afrique, c'est « the sweet gangrenous smell of certain leprous skins » (p. 18), l'ex-architecte faisant lui-même partie du groupe des «lépreux ». Le Fleuve a pris une forme circulaire et, quant à la Route, cet axe de pénétration où avait triomphé la fierté coloniale, elle est désormais "narrow like a coffin or a grave » (p. 30). Dans les ténèbres extérieures («darkness outside », p. 176), derrière le «green jungle wall» (p.26) qui, comme chez Cornélus, est animé et "s'approche", "there was little in the forest to appeal to the romantic. It was completely empty. It had never been humanized, like the woods of Europe » (p. 54).

Comme pour illustrer d'avance les hypothèses de Piniau, Greene (qui lui-même ne semble pas toujours bien informé, comme à propos des albinos) met d'ailleurs en scène un personnage de journaliste racontant n'importe quoi, pourvu que cela soit sensationnel ("They won't know the bloody difference »), évoquant Stanley mais selon une phraséologie conradienne («The eternal forest broods along the banks unchanged since Stanley and his little band»), et titrant son article, de manière éloquente : "The White Man's Grave»(p.97-98). Toutefois, cette opinion de Parkinson, largement partagée par d'autres personnages du roman, colonialistes qui ne demandent qu'à rentrer en Métropole et qui « haïssent ce pays », n'est pas celle de Querry, ni sans doute de Greene : c'est du tombeau de l'homme tout court, ou plus précisément du sujet historique tel qu'il a été développé en Occident dans une perspective de progrès pour le monde, qu'il s'agit ici : la fin du régime colonial est une métaphore.

D'un point de vue plus théorique, la suggestion de Piniau à propos du renoncement au projet historique est de nature à modifier le sens de l'adjectif « lointain » : en réalité, la distance physique importe peu, et en elle-même la distance pratique (les difficultés de

26 GreEnE (Graham), A Burnt-Out Case. (1960). London, Penguin Books, 1975, p.16 ; La Saison des pluies. Roman traduit de l'anglais par Marcelle Sibon. Paris, Laffont, 1961 ; coll. Presses Pockets n²175, 1991, p.28. 
communication) ne suffit pas à expliquer comment et pourquoi toute une sphère du donné humain bascule dans l'altérité. Est « lointain » ce qui est senti comme autre, ce qui, pour une raison ou pour une autre, est altérifié, donné comme inaccessible et par là pourvu de supposés pouvoirs mythiques. Est «lointain» ce qui, en d'autres termes encore, sort du champ de ma responsabilité, ne m'apparait plus comme un Visage, mais comme le masque grimaçant de quelque pulsion ou de quelque divinité (Greene, de ce point de vue, exprime une position très influencée par l'existentialisme, mais aussi par le christianisme; sur ce point, il s'éloigne de la tradition conradienne). On conçoit aussi, sur cette base, l'indifférence relative du média emprunté (cinéma, roman, littérature canonique ou Trivialliteratur). On comprend enfin pourquoi la langue est relativement non relevante: au-delà de différences socio-historiques caractérisant chaque pays colonisateur (ou auxiliaire de pays colonisateurs, comme les pays scandinaves), l'imaginaire occidental est relativement unifié par un complexe identitaire commun, marqué par le développement et l'extension, mais aussi par le sanglot de l'homme blanc, le remords, la conscience du péché ; par le récit faustien, en somme, qui projette sur une figure diabolique autre à la fois sa volonté de puissance et son désir d'en être puni.

Dès lors s'éclaire aussi l'opposition exotisme vs antexotisme: si la tradition conradienne désespère qu'une action change jamais l'Histoire, celle de Stanley appartient au contraire à l'Histoire en marche. Lorsqu'un romancier africain comme Bolya choisit de se ranger dans la première, il serait assez vain de lui reprocher un manque d'information ou l'adhésion naïve à un fantasme européen à propos de l'Afrique centrale; le soupçon qu'il ait voulu rejoindre une sensibilité esthétique plus conradienne que stanleyienne sur le marché européen et certainement plus valorisante sur le marché de la légitimation littéraire «moderne » procède déjà d'une analyse plus fine ${ }^{27}$. Mais l'essentiel est de voir qu'un tel choix inscrit une désespérance historique en définitive plus dommageable pour l'Afrique que l'imagerie qui sert à son expression. Et que ce choix fait le jeu du primitivisme ${ }^{28}$, cette version coloniale-ethnologique de l'afro-pessimisme, toujours prompt à assigner l'Afrique à quelque irrationalité, dont peu importe qu'elle soit attendrissante ou, comme ici, « barbare ».

\section{Dans la clôture?}

27 Ceci est de nature à expliquer en partie l'écart rhétorique qui sépare le corpus littéraire des exilés congolais zaïrois de celui des écrivains restés au pays ; cf. les discussions qui affleurent dans Littératures du Congo-Zaïre. Actes du colloque international de Bayreuth (22-24 juillet 1993). Réunis par Pierre Halen et János Riesz. Amsterdam-Atlanta, Rodopi, 1995, XIV+424 p. [pp.1318], (= Matatu, $\left.\mathrm{n}^{\circ} 13-14,1995\right)$. et la question nationale», dans Cahiers d'études africaines, 121-122, XXX-1-2, 1991, pp.191213. 
La position d'un Naipaul, dans une telle configuration, ne pouvait manquer d'être particulière. Le protagoniste de $A$ Bend in the River ${ }^{29}$, issu des communautés indiennes établies sur la Côte Est de l'Afrique, ne se sent guère d'affinités, sans doute, avec le discours colonialiste du «Fardeau de l'Homme blanc», jugé hypocrite (on ne lui reproche forcément ni l'extranéité ni l'exploitation, mais la dissimulation de l'exploitation sous un voile civilisateur), pas plus qu'avec son envers, celui du «Sanglot» tiers-mondiste. C'est d'un point de vue pragmatique, lié à la survie matérielle, qu'il regrette la "paix coloniale » dont la fin a aussi marqué la dislocation de sa communauté. Il reprend, à défaut de mieux, une boutique vacante à StanleyvilleKisangani, mais il ne s'y établit pas vraiment, se gardant d'habiter véritablement un lieu auquel il demeure étranger. Il laisse ainsi inchangé, et jusqu'à un certain point inhabité, l'appartement qu'il a « repris » à une curieuse locataire belge. La déglingue progressive de l'État zaïrois rend sa situation de plus en plus intenable : en fin de compte, il est donc logiquement expulsé de ce lieu qu'il a observé, sans doute, autant que possible avec un regard antexotique, mais que menacent comme une lèpre un certain nombre de sèmes conradiens: l'encerclement, l'immobilité historique, l'impossibilité d'agir ou même de se maintenir, l'opacité progressive du paysage.

La perception naturaliste de la déglingue et du marasme engendrés par la politique mystérieuse du « Grand Homme »-qu'on a pourtant tenté de comprendre - ajoute à ce tableau de l'impavidité une dimension de déchéance : le motif récurrent de la ruine, et plus encore celui des jacinthes d'eau entrainées par le fleuve vers le néant, illustrent l'abandon de la lutte historique, tentée à une très modeste échelle par l'immigré. À cet égard, on regrettera que la traduction française ait eu recours au terme de "rapides », alors que celui de «chutes » eût mieux traduit mieux l'ambivalence de l'anglais Falls pour désigner la configuration du Fleuve à cet endroit. On retrouve, dans $A$ Bend in the River, comme chez Greene, le thème de l'architecture inutile et, comme dans Le Reste $d u$ monde, le tableau d'un érotisme aussi exacerbé que sans issue (avec une Blanche). On pourrait multiplier les points de convergence ${ }^{30}$.

La position de Naipaul est sans doute spécifique : ni européen ni africain, son personnage échappe au fantasme du prométhée comme lui est étranger le remords faustien. Il ne vient pas en Afrique centrale à la recherche de quelque Autre d'ordre moral, culturel ou métaphysique. Homme du tiers-monde lui-même, il sympathiserait sans doute volontiers avec les destinées d'un continent auquel il appartient par son histoire ; et néanmoins il n'y parvient pas : ce pays s'avère rétif dès le voyage qu'il entreprend pour pénétrer en son sein. Le propos dès lors se partage entre un pragmatisme antexotique qui cherche à comprendre tout en demeurant sur la réserve, et, de plus en plus, un exotisme contraint par le Réel. Pour une part, il s'agit sans doute de la projection du sentiment que sa propre collectivité est condamnée par l'Histoire à

NAIPAUL (V.S.), A Bend in the River (1979). Harmondsworth, Penguin Books, 1980, 287 p. ; tr. fr. A la courbe du fleuve. Trad. de l'anglais par Gérard Clarence. Paris, Albin Michel (1982), coll. 10/18 - Domaine étranger, 1995, $381 \mathrm{p}$. 
mourir comme telle mais, pour une autre part, il constitue aussi une interrogation devant l'incompréhensible anéantissement de l'Histoire elle-même, en ce lieu.

Ce tiers observe l'Afrique contemporaine en son centre géographique mais aussi peut-être en son centre symbolique (dans la mesure où, le fantôme de l'ex «perle du Congo » hantant ces lieux, l'inversion de la notion même de progrès s'y fait dès lors plus remarquablement sentir). Et, renonçant à expliquer cet anéantissement ou ne lui trouvant pas d'explication suffisante, il ne trouve pour la dire néanmoins que des formules mythifiantes : "It was unnerving, the depth of that African rage, the wish to destroy, regardless of the consequences» (p. 32). Ce sont des populations qui ne peuvent vivre que sous le signe de la « proie»:

But what happened was not new. People who had growned feeble had been physically destroyed. That, in Africa, was not new ; it was the oldest law of the land (p.35).

It was in the history of the land : here men had always been prey. [...] The people here were malins the way a dog chasing a lizard was malin, or a cat chasing a bird. The people were malins because they lived with the knowledge of men as prey (p.60).

Le recours à l'adjectif français par les personnages est à mettre en rapport non pas avec l'idée d'astuce ou de ruse, mais avec l'anglais malice, qui figure dans le contexte et que le traducteur français fait bien de ne pas rendre par le faux-ami malice, mais par le terme de méchanceté : il s'agit bien de malveillance, d'intention criminelle délibérée. Nous ne sommes pas loin d'une diabolisation, d'autant moins compréhensible qu'elle est reportée sur une collectivité anonyme, à peu près indistincte ${ }^{31}$.

La volonté historique, même modeste, doit s'annihiler dans ce pays dont les habitants «were like people who didn't know their own mind"; ils ont certes des circonstances atténuantes: "They had suffered so much», et pour un peu, on les prendrait en pitié. Mais à cette explication-justification humaniste, quelque chose résiste, un quelque chose qui dépasse la compréhension : "But it was people like them, going back to their villages, who wished to lay the town low again. Such rage!» (pp.72-73). Going back: l'opposition village-ville, purement sociologique au début du roman, devient vite culturelle et chronologique. D'autant que la Ville s'était autrefois donné un projet de métissage 32 auquel le personnage comme l'auteur post-colonial ne pouvaient qu'être particulièrement sensibles. Le roman de Naipaul enregistre en réalité la désuétude ou l'impertinence de certaines problématiques, comme celle du nationalisme, qui semblent un horizon purement idéel eu égard à ce qu'il faut bien appeler la déchéance d'un lieu concret voué à la ruine ${ }^{33}$. À cet égard, le hiatus qui

31 Sauf l'individu Ferdinand qui, après avoir représenté la jeunesse incertaine, dira le désarroi du responsable administratif pris dans le système et impuissant à le modifier.

32 Ce projet est plusieurs fois rappelé par la devise latine : «Miscuique probat populos et foedera jungi», qui figure encore au bas du monument détruit, autrefois érigé à l'occasion des 60 ans de la ligne de transport fluvial. in the Africa of words ? Did anyone on the Domain believe ? Wasn't the truth what we in the town lived with [...] ?» (p.130). 
sépare le Domaine, construit sous le signe de l'artifice, de l'agglomération urbaine (qui n'est plus, qui est de moins en moins une Ville), marque dans l'espace ce qui, en termes d'espérance historique, est suspendu.

Life in our town was arbitrary enough. [...] in the town, where all was arbitrary and the law was what is was, all our lifes were fluid. We none of us had certainties of any kind. Without always knowing what we were doing we were constantly adjusting to the arbitrariness by which we were surrounded. In the end we couldn't say where we stood (p.198).

L'absence de loi concerne à la fois la vie sociale, la perception des choses et la morale ; d'où le constant répété de Maresh: "It isn't that there's no right and wrong here. There's no right» (ib.). Si le paysage s'abolit dans l'enfermement des monceaux d'ordures et du fleuve dont la présence se fait sentir all around (p.130; «à la ronde ", traduit G. Clarence), le petit mot here est pour limiter la portée du constat de perdition à une zone déterminée de la planète. La perdition lui est-elle consubstantielle ou s'explique-t-elle malgré tout par une série de décisions erratiques qu'il sera toujours possible de rectifier par d'autres décisions plus rationnelles et plus logiquement orientées vers un mieux-être ? Le roman ne tranche pas, jouant plutôt à la fois des deux dimensions mythique et historique. Il tranche pourtant quelque chose tout à la fin du roman, et dans le sens du désespoir pour les populations locales: les attaches qui permettaient à la barge africaine d'être tirée ou poussée ${ }^{34}$ par le steamer sont rompues à la suite d'une attaque armée qui rappellerait lointainement celles subies par Stanley sur le fleuve, si ses acteurs n'étaient désormais indiscernables. Et, tandis que le narrateur et quelques privilégiés continuent le voyage qui leur permettra de s'en sortir, la barge dérive dans la nuit au milieu des jacinthes flottantes, sortant de la «lumière blanche » que le projecteur du steamer dardait encore sur son destin. Si l'Univers entier n'est donc pas nécessairement concerné par la scène conradienne comme il l'était chez Geramys, le pays de prédilection où elle pouvait littérairement se jouer semble assigné à n'être jamais que celui de "slave peoples [...] physically wretched, half men in everything except in their capacity to breed the next generation » (p. 81).

Mais l'entremêlement même des deux dimensions mythique et historique, des deux paradigmes conradien et stanleyien, dans l'ensemble du roman, est pour démentir ce que de tels propos semblent avoir de définitif. Et, si l'on en croit Piniau, il ne s'agirait que d'en revenir à une position d'initiative historique, donc aussi à ses conditions de possibilité socio-politique, pour que ce qui, ici, chute ou rechute dans une nature supposée redevienne à la fois Paysage et Visage. Reste la question des conditions de possibilité littéraires: pour associer modernisme, humanisme et progressisme, il y faudrait le génie d'un Verhaeren (idéologiquement fort proche, soit dit en passant, de la position d'un de Lichtervelde que nous avons citée). La question pourrait être en définitive de savoir si une œuvre procédant de l'antexotisme peut s'imposer comme «moderne », et sur quel marché de la reconnaissance. C'était déjà la question qui séparait, dans les années 1920, le colonial Joseph-Marie Jadot, plus attentif à une

34 «The passenger barge was not towed behind theses days -- that was now considered a colonial practice. Instead, the barge was lashed to the forward part of the steamer» (p.286). 
certaine morale littéraire lorsqu'il s'agissait du Congo, du métropolitain Gaston-Denys Périer, découvreur de Conrad et surtout soucieux de valorisation esthétique : à cet égard, le rôle que jouera à l'avenir l'institution littéraire au Congo-Zaïre même sera sans nul doute déterminant. 